\title{
Effects of Health Education on Cardiovascular System in Middle- aged and Elderly People-Take Aerobic Exercise for Example
}

\author{
Hai-Jun Zhang ${ }^{1 *}$, Xiao-Tao Guo ${ }^{1}$, Hai-Li Zhang ${ }^{2}$ \\ ${ }^{1}$ Hunan First Normal University, CHINA \\ ${ }^{2}$ Hunan University of Arts and Science, CHINA
}

Received 9 June 2017 - Revised 17 September 2017 - Accepted 20 October 2017

\begin{abstract}
Our country has entered the aged society, the health education in the elderly is imminent. The studies in the effects of aerobic exercise mode on the cardiovascular system in the middle-aged and elderly people will provide theoretical basis and practice mode. Research methods: 30 healthy people (aged 50-70) were selected to undertake 8-week exercise as the aerobic exercise model (45mins/day, 5times/week). Measured indexes such as cardiovascular functions, hemodynamics flow, blood fat and renin-angiotensin-aldosterone system (RAAs) before and after the exercise, and then analyzed and compared them by mathematical statistics. Research result: 1) Cardiovascular function indexes pulse rate (PR), pulse pressure(PP), systolic pressure(SP), diastolic pressure(DP), valid pump effort(VPE) of left heart, heart oxygen consumption volume (HOV) decrease more or less, while cardiac output(CO), stroke volume(SV), stroke index $(\mathrm{SI})$, cardiac index $(\mathrm{Cl})$ is obviously higher than before $(p<0.01)$. 2) There is no significant difference in hemorrheology indexes like thrombus formation lime (TFL), microcirculation strees time (MST), and erythrocyte aggregation index(VAI), while blood viscosity (nb), blood reduced viscosity ( $\eta r)$, fibringen (PFC), plasma viscosity $(n p)$, hematocrit (Hct) drop apparently $(p<0.05)$. 3) Blood-lipoid indexes cholesterol $(\mathrm{Ch})$, triglyceride $(\mathrm{Tg})$, low density lipoprotein (LDL) gets lower after the experiment, while high density lipoprotein (HDL) is higher $(p<0.01)$. 4) Angiotensin I (Ang I) is obviously lower than that before the experiment, but angiotensin II (Ang II) and aldosterone (Ald) has no significant differences. Research Conclusion: the aerobic exercise model of jogging plus broadcast gymnastics is beneficial for middle-aged and elderly people 1) improve their cardiovascular system function. 2) decrease the blood viscosity with a certain significance to prevent high blood viscosity syndrome (HVS) for them. 3) prevent the coronary atherosclerosis in them. 4) accelerate renal blood flow in them, reduce angiotensin I secretion, improve cardiovascular functions, strengthen cardiovascular compliance, prevent and reverse the risk of cardiovascular disease.
\end{abstract}

Keywords: health education, aerobic exercise, middle-aged, aged people, cardiovascular system

\section{INTRODUCTION}

The core of health education is to educate people to build up the health consciousness, urges people to change unhealthy behaviors and life style, and develops good life style to reduce or eliminate the risk factors affecting health (Mustafa G., Rumeysa D., Bilgen B. 2017). Sports health education is an important part of health education. However due to the little scientific studies on the relationship of the physiological and motion attributes (including events, time, intensity, location), education is also rare. Which causes many people exercise less, or blindly exercise, not only failed to reach the purpose, but also the opposite effects. With the whole world stepping the old people's society, sports health education has become an important problem that many countries and people need to solve urgently (Fan K. K., Xiao P. W. and Su C.H. 2015). 


\section{Contribution of this paper to the literature}

- Aerobic exercise can improve cardiovascular functions, strengthen cardiovascular compliance, prevent and reverse the risk of cardiovascular disease.

- Aerobic exercise has significance for preventing high blood viscosity syndrome (HVS) for middle-aged and aged people.

- $\quad$ Aerobic exercise can prevent diseases such as coronary atherosclerosis, hypertension and heart disease.

Aerobic exercise refers to the exercise that is undertaken in the circumstances of adequate oxygen supply. Its basic character is that the supply and demand of oxygen is equal, thus to reach physiological balance (WANG Zheng-zhen \& ZHAO Hui-juan.2005) Jogging and free-standing exercises are all the most common aerobic exercises as well as the most commonly undertaken and favorite exercises for the middle-aged and elderly people. However, they are more beneficial for just one particular part of your body both on health and exercise, and it's boring and dull to do one single sport. Apparently, an all-body exercise filled with fun is more attractive and much healthier than a single one. Cardiovascular diseases are common among middle-aged and aged people (MA Zhi-jun, 2010), which are harmful for people's health, and even the killer for those people. Like hypertension is the major cardiovascular risk factor that caused the increase of mortality (Chobanian AV, Bakrris GL \& Black HR,et al,2003), affecting nearly one billion people worldwide. It is people's common aspiration to keep cardiovascular system healthy and enhance cardiovascular immunity. Undoubtedly, it's very important to study the effect of an aerobic exercise model on cardiovascular system in middle-aged and elderly people, and strengthening the sports health education for them.

The following research questions are explored in this study:

1. How and to what extent does the Aerobic exercise effect on the Cardiovascular System Function?

2. How and to what extent does the Aerobic exercise effect on the Hemorheology Indices?

3. How and to what extent does the Aerobic exercise effect on the Blood Lipid?

4. By what way does the Aerobic exercise Prevent Cardiovascular Diseases?

\section{RESEARCH DESIGN AND APPROACH}

Select 30 healthy volunteers aged 50 to 75 (12 males, 18 females) from Yuelu District in Changsha City as the objects. On inquiring and testing, all of them don't have contraindication to exercise.

Warming-up, undertaken on 20 August 2016, five times per week, began in six pm per day. At first, the participants keep running for 20-minute and aerobic gymnastic with the exercise intensity of 50\% maximal heart rate (220-age)( LI Xiu-li,2003), then increased 3-5 minutes exercising time and intensity every day mainly by fastening running stride and the rhythm of aerobic gymnastic. The experiment really started on 1 September and took place during $6 \mathrm{pm}$ to $7 \mathrm{pm}$ every day. The aerobic exercise mode was 15 minutes jogging +5 minutes joint and ligaments motion +15 minutes setting-up exercises of "the energy of youth" +5 minutes relaxation running and tapping movement. Heart rate of the jogging and setting-up exercises had to reach $60 \%-75 \%$ of the HR max (MA Zhi-jun,2010; LI Xiu-li,2003; ZHOU Li-ying, WANG Xin \& HONG Le-feng, et al,2008; YU Wan-bing,2008) The participants did the exercise for 8 weeks, 5 times a week and were measured indexes before and after the experiment.

Cardiovascular function indexes: pulse rate (PR), pulse pressure(PP), heart oxygen consumption volume $(\mathrm{HOV})$, stroke volume(SV), cardiac output(CO), cardiac index (CI), stroke index(SI), systolic pressure(SP), diastolic pressure (DP), valid pump effort(VPE) of left heart, effect work of Kinetics (EWK), arterial compliance(AC) etc.

Hemorheology indexes: fibrinogen (PFC), hematocrit (Hct), blood viscosity

$(\eta b)$, blood reduced viscosity ( $\eta r)$, plasma viscosity ( $\eta p)$, microcirculation stress time (MST), erythrocyte aggregation index(VAI), hrombus formation lime (TFL) etc.

Blood-lipoid indexes: cholesterol (Ch), triglyceride (Tg), high density lipoprotein (HDL), low density lipoprotein (LDL).

Renin angiotensin aldosterone system indexes: Angiotensin (Ang I), angiotensin II (Ang II), aldosterone (Ald).

Test Instruments and Reagents: Three-leads electrocardiograph AT-1, RGZ-RT weight scale, and Mindray BS400 automatic biochemistry analyzer by Contec Medical Systems Co., Ltd, AZN-IIIA cardiovascular function examination apparatus by Institute of Intelligent Machines, Chinese Academy of Sciences.

Date Processing: Adopting the software SPSS to process the detection parameters and the result is represented in average \pm standard deviation. The difference between groups were analyzed by test, and the significance level is $\mathrm{p}<0.05$. 
Table 1. Changes of cardiovascular function before and after the aerobic exercise model experiment

\begin{tabular}{|c|c|c|c|c|c|c|c|c|c|c|}
\hline Index & $\begin{array}{c}\text { PR } \\
\text { (beat/min) }\end{array}$ & $\begin{array}{c}\text { LVWI } \\
\left(g^{\prime} \mathrm{m} / \mathrm{beat}^{2} / \mathrm{m}^{2}\right)\end{array}$ & VPE (kg/beat) & $\begin{array}{c}\text { HOV } \\
(\mathrm{ml} / \mathrm{min})\end{array}$ & SP $(\mathrm{mmHg})$ & $\mathrm{DP}(\mathrm{mmHg})$ & PP $(\mathrm{mmHg})$ & $\begin{array}{c}\text { CO } \\
(\mathrm{L} / \mathrm{min})\end{array}$ & SV (ml /beat) & $\begin{array}{c}\text { SI } \\
\left(\mathrm{ml} / \mathrm{beat} / \mathrm{m}^{2}\right)\end{array}$ \\
\hline \multirow{2}{*}{ Before } & $77.74 \pm$ & \multirow{2}{*}{$74.319 \pm 16.65$} & $1.818 \pm$ & $43.563 \pm$ & \multirow{2}{*}{$132.44 \pm 21.852$} & $78.08 \pm$ & $53.462 \pm$ & $6.10 \pm$ & $80.57 \pm$ & $52.592 \pm$ \\
\hline & 9.56 & & 0.44 & 9.521 & & 12.24 & 15.106 & 1.201 & 11.18 & 9.366 \\
\hline \multirow{2}{*}{ After } & $72.80 \pm$ & $70.07 \pm$ & $1.725 \pm$ & $40.54 \pm$ & \multirow{2}{*}{$127.09 \pm 19.111$} & $74.85 \pm$ & $50.257 \pm$ & $6.65 \pm$ & $84.79 \pm$ & $55.673 \pm$ \\
\hline & 9.32 & 15.82 & 0.30 & 6.06 & & 10.73 & 13.360 & $1.033^{*}$ & $15.99^{*}$ & $7.54^{\star}$ \\
\hline Index & $\begin{array}{c}\mathrm{Cl} \\
\left(\mathrm{L} / \mathrm{mim} / \mathrm{m}^{2}\right) \\
\end{array}$ & EWK & $A C$ & FEK & BV & MRT & $\begin{array}{c}\text { TPR } \\
\text { (dyne's/cm5) } \\
\end{array}$ & $\begin{array}{c}\text { PAWP } \\
(\mathrm{mmHg}) \\
\end{array}$ & $\operatorname{PAP}(\mathrm{mmHg})$ & $\begin{array}{c}\text { PAR } \\
\text { (dyne's/cm5) } \\
\end{array}$ \\
\hline Before & $\begin{array}{c}4.314 \pm \\
0.709 \\
\end{array}$ & $\begin{array}{c}0.315 \pm \\
0.039 \\
\end{array}$ & $\begin{array}{c}1.610 \pm \\
0.496 \\
\end{array}$ & $\begin{array}{c}0.3101 \pm \\
0.037 \\
\end{array}$ & $\begin{array}{c}4.538 \pm \\
0.881 \\
\end{array}$ & $\begin{array}{c}14.953 \pm \\
2.555 \\
\end{array}$ & $\begin{array}{c}901.454 \pm \\
276.247 \\
\end{array}$ & $15.310 \pm 5.703$ & $21.355 \pm 7.899$ & $\begin{array}{c}217.463 \pm \\
109.22 \\
\end{array}$ \\
\hline After & $\begin{array}{l}4.606 \pm \\
0.611^{*} \\
\end{array}$ & $\begin{array}{c}0.341 \pm \\
0.049 \\
\end{array}$ & $\begin{array}{c}1.683 \pm \\
0.519 \\
\end{array}$ & $\begin{array}{c}0.3216 \pm \\
0.2974 \\
\end{array}$ & $\begin{array}{c}4.904 \pm \\
0.832 \\
\end{array}$ & $\begin{array}{c}15.203 \pm \\
2.647 \\
\end{array}$ & $\begin{array}{c}905.125 \pm \\
278.122 \\
\end{array}$ & $15.157 \pm 5.846$ & $21.607 \pm 6.870$ & $\begin{array}{c}222.475 \pm \\
90.81 \\
\end{array}$ \\
\hline
\end{tabular}

Table 2. Changes of hemorrheology indexes before and after the aerobic exercise experiment

\begin{tabular}{cccccccccc}
\hline Index & Hr/mps-s-1 & PFC/g·L-1 & Hp & MST/s & VAl & TFL & HCT/VOL & Hb \\
\hline \multirow{2}{*}{ Before } & $7.55 \pm$ & $2.84 \pm$ & $1.86 \pm$ & $23.8 \pm$ & $0.72 \pm$ & $0.85 \pm$ & $0.61 \pm$ & $3.93 \pm$ \\
& 0.97 & 0.63 & 0.12 & 0.75 & 0.49 & 0.13 & 0.05 & 0.66 \\
\hline \multirow{2}{*}{ After } & $6.99 \pm$ & $2.61 \pm$ & $1.60 \pm$ & $24.14 \pm$ & $0.66 \pm$ & $0.72 \pm$ & $0.42 \pm$ \\
& $0.82^{*}$ & $0.87^{\star}$ & $0.18^{\star}$ & 0.68 & 0.32 & 0.17 & $0.05^{\star}$ & $0.57^{\star}$ \\
\hline
\end{tabular}

Table 3. Changes of blood-lipoid indexes before and after the aerobic exercise experiment

\begin{tabular}{ccccc}
\hline Index & $\mathbf{C H}$ & TG/mmol·L-1 & LDL/mmol/L-1 & HDL-C/ mmol·L-1 \\
\hline Before & $5.38 \pm 1.52$ & $1.20 \pm 0.53$ & $2.37 \pm 0.26$ & $1.26 \pm 0.27$ \\
\hline After & $4.55 \pm 1.25^{*}$ & $0.81 \pm 0.52^{*}$ & $2.14 \pm 0.14^{\star}$ & $1.48 \pm 0.29^{\star}$ \\
\hline
\end{tabular}

Table 4. Changes of renin angiotensin aldosterone system indexes before and after the aerobic exercise experiment

\begin{tabular}{cccc}
\hline Index & Ang I / $\mathbf{m g} \cdot \mathbf{m L}-\mathbf{1} \cdot \mathbf{h}-\mathbf{1}$ & Ang I /pg·mL-1 & ALD/pg·mL-1 \\
\hline Before & $2.52 \pm 0.91$ & $83.6 \pm 16.8$ & $160.6 \pm 85.3$ \\
\hline After & $0.51 \pm 0.84^{*}$ & $97.5 \pm 20.08$ & $171.9 \pm 70.4$ \\
\hline
\end{tabular}

\section{RESULTS}

The Changes of Cardiovascular Function Indexes: Compared the cardiovascular function indexes before and after the experiment, pulse rate (PR), pulse pressure (PP), consumption volume (HOV), left ventricular work index (LVWI) decreased more or less; stroke volume (SV), cardiac output (CO), cardiac index (CI), stroke index (SI), were obviously higher than before $(\mathrm{p}<0.01)$; there was no significant difference in effect work of Kinetics $(\mathrm{EWK})$, arterial compliance (AC), effective blood volume (BV), artery elasticity count (FEK), total peripheral resistance (TPR), pulmonary vascular resistance (PVR), pulmonary artery wedge pressure (PAMP), pulmonary artery pressure (PAP) $(\mathrm{p}>0.05)$ (see Table 1).

The Changes of hemorrheology indexes: Compared hemorrheology indexes before and after the experiment, no significant difference can be observed in thrombus formation lime (TFL), microcirculation strees time (MST),

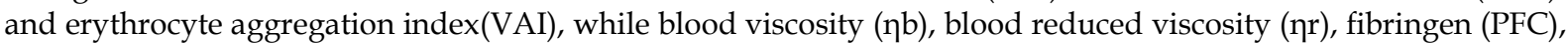
plasma viscosity $(\eta p)$, hematocrit (Hct) had great changes $(p<0.05)$ (see Table 2$)$.

The Changes of Blood-lipoid Indexes:Compared blood-lipoid indexes before and after the experiment, we found that cholesterol (Ch), triglyceride (Tg), low density lipoprotein (LDL) were lower after the experiment, while high density lipoprotein (HDL) was higher $(\mathrm{p}<0.01)$ (see Table 3).

The Changes of Renin Angiotensin Aldosterone System Indexes: Angiotensin I (Ang I) was obviously lower than that before the experiment, but angiotensin II (Ang II) and aldosterone (Ald) had no significant differences (see Table 4).

\section{DISCUSSION AND CONCLUSION}

The Aerobic Exercise Model of Jogging + Broadcast Gymnastics can Promote Cardiovascular System Function: The experiment proves that the aerobic exercise model of jogging + broadcast gymnastic can effectively improve cardiovascular system function, especially in toning the walls of veins and preventing arteriosclerosis of middleaged and elderly people, which is the same with the study result directed by GAO Lin-Zhou et al (1997). The mechanism is that aerobic exercise can regulate the balance of central nervous system between excitation and inhibition, especially can improve the autonomic nervous system function, shown as the reinforcement of 
inhibitory (Wang SongTao, Ceng YunGui \& Wang AnLi, 2007). Apart from the improvement of the cerebral cortex and respiratory and urinary system, aerobic exercise also benefits the cardiovascular system, strengthens vasodilatation and vasoconstriction functions so that the elasticity of blood vessels can be improved (Yu LAN, 2002). Moreover, aerobic exercise helps dilate blood vessels, leading to the fall of blood pressure and the reduction of peripheral resistance, which slows down the decreasing tendency of the blood-flow in middle-aged and elderly people, makes each organ get more blood, and effectively enhance the cardiac contractility (Perini R, Fisher N \& Veicsteinas A, et al,2002). Aerobic exercise can make tissue cells get better exchange of material because of the increase in vascular wall area (GENG Tong-man, 1992). It also can delay the decline of artery dispensability and the rise of pulmonary vascular resistance which come along with age, thus to reduce the preload and afterload of heart, decline the myocardium oxygen consumption in load exercise and the cardiovascular activity is even economical, finally to reach the goal of retarding cardiovascular function aging and effectively preventing and curing cardiovascular diseases (Yang Qian, SuiBo \& Hu YunLian, et al, 1996). From the experimental results, as blood moves through tissue, the extension of microcirculation residence time (MRT) and microcirculation strees time (MST) makes the speed of blood flow slow down, so that the tissue cells can do a more effective interchange of material. According to statistics, middle-aged and elderly people developing good life habits, and actively participating in sports can reduce the cardiovascular risk of $64 \%$. Aerobic exercise is the first choice for middle-aged and elderly people in sports health. They should be educated more about aerobic exercise knowledge and trained their ability to exercise themselves (Marcia C. Linn, Bat-Sheva Eylon, Anna Rafferty, Jonathan M. Vitale, 2015).

The Aerobic Exercise Model of Jogging + Broadcast Gymnastics can Improve Hemorheology Indices: The experiment results show that: no significant difference can be observed in thrombus formation lime (TFL), microcirculation strees time (MST), and VAI, while blood viscosity ( $\eta \mathrm{b})$, blood reduced viscosity ( $\eta \mathrm{r})$, fibringen $(\mathrm{PFC})$, plasma viscosity (np), hematocrit $(\mathrm{Hct})$ had great changes $(\mathrm{p}<0.05)$. We often hold that the blood of elderly people becomes sticky, dense, agglomerate, and collective with ages, which is called clinically as high blood viscosity syndrome (HVS). Many common and frequently-occurring diseases in elderly people are related to it (Yang Qian, SuiBo \& Hu YunLian, et al, 1996). But we should realize that low blood viscosity can lead to various diseases such as anemia and malnutrition, so the appropriate hemorheology indices are seen as a sign of health in middle-aged and elderly people. This research shows that the indexes reflecting hypercoagulability and hyperviscosity decreased, which proves the benefits of aerobic exercise mode to middle-aged and elderly people.

Aerobic Exercise Model of Jogging + Broadcast Gymnastics can Improve Blood Lipid: Coronary heart disease and arteriosclerosis these diseases also frequently occur in middle-aged and elderly people and attribute to hyperlipidemia. The main lipid cholesterol (Ch) and triglyceride (Tg) in serum is the key indexes reflecting Coronary heart disease and arteriosclerosis (Liu ZongLiang, 1993). Many previous studies indicate that the improvement of blood lipid levels caused by exercise has an important relationship with enzyme, for example, exercise can improve the level of lipase in rats, and the reason is that the organism will accelerate decomposition of LDL-C and CM by increasing the level of LPS in blood (Guo YingJie, 2005) : Aerobic exercise also can raise the level of HDL2 by increasing the activity of lecithin-cholesterol acyltransferase (LCAT); and under the condition of highfat diet, it makes the receptor of low-density lipoprotein mRNA can't be affected (FuLi, 1996; Chen JiDi, 2002). Some researchers found that aerobic exercise can cause significant decrease in the level of ApoB and increase in the level of ApoA I, thus resulting in the decrease of LDL and the increase of HDL (Cui YouQiong \& Wu HongYing, 2010). LPL is not only the key enzyme in metabolism of lipoprotein, but also plays an important role in promoting the transition from LDL to HDL. Besides, aerobic exercise can increase HOL and decrease Tg by improving LPL's activity (Seip RL, Angelopoulos TJ \& Semenkovich CF, 1995).

The experiment results prove that cholesterol (Ch), triglyceride (Tg), low density lipoprotein (LDL) were lower after the experiment, while high density lipoprotein (HDL) was higher, which showed that aerobic exercise can effectively improve lipid metabolism rate and levels of $\mathrm{HDL}$, and lower the concentration of $\mathrm{Ch}, \mathrm{Tg}$, and LDL, thus to prevent various diseases like coronary atherosclerosis, hypertension, heart disease, and cerebral arteriosclerosis.

Aerobic Exercise Model of Jogging + Broadcast Gymnastics can Prevent Cardiovascular Diseases: The experiment results show: After eight weeks of aerobic exercise, Ang I was obviously lower than that before the experiment, which proved that aerobic exercise can fast renal blood flow, makes renal arterioles get a rich blood supply and reduces the secretion of renin and Ang I. All these show that the aerobic exercise model can promote cardiovascular function, strengthen cardiovascular compliance, prevent and reverse the risk of cardiovascular disease. 


\section{REFERENCES}

Chen, J. (2002). Aerobic-exercise, Gene Expression and Chronic Diseases. The Journal of Chinese Sports, 21(1), 61-65.

Chobanian, A. V., Bakris, G. L., Black, H. R., Cushman, W. C., Green, L. A., Izzo, J. L. Jr, Jones, D. W., ..., Roccella, E. J. (2003). The seventh Report of the Joint Committee on Prevention, Detection, Evaluation, and Treatment of High Blood Pressure. Hypertension, 42, 1206-52.

Cui, Y. Q., Wu, H. Y., \& Duan, X.-H. (2010). On The Changes of Different Intensive TaijiQuan Practice on Blood Biochemical Indications. Modern Preventing medicine, 37(20), 3881-3883.

Fan, K. K., Xiao, P. W., \& Su, C. H. (2015). The Research and Evaluation of Drug-use Habits of People in North Cyprus. EURASIA Journal of Mathematics, Science and Technology Education, 11(5), 1211-1229. doi:10.12973/eurasia.2015.1413a

Fu, L. (1996). On the Mechanism of Aerobic-exercise preventing high-lipid. Beijing: Beijing medical university.

Gao, L.-Z., \& Tang, A.-L. (1997). The Analysis of the Effects of Taijiquan on Cardiovascular Function in Elders. ACTA Academiae Medicinae Bengbu, 22(6), 436-437.

Geng, T.-M. (1992). The Effects of Sports on the Function of cardiovascular - Investigation on the seniors and middle aged of our institute. The Journal of Foreign affairs College, 02, 67-69.

Guo, Y. (2005). On Effects and Mechanism of Pyruvate added on Players 'Body Composition and Lipid Metabolism (Doctoral Thesis). Beijing University.

Gürman, M., Demirdamar R., \& Basgut, B. (2017). The Research and Evaluation of Drug-use Habits of People in North Cyprus. EURASIA Journal of Mathematics, Science and Technology Education, 13(3), 791-801. doi:10.12973/eurasia.2017.00643a

Li, X.-L. (2003). The Biological Analysis of Aerobics. Journal of Beijing University of Physical Education, 26(6), 776-778.

Linn, M. C., Eylon, B.-S., Rafferty, A., \& Vitale, J. M. (2015). The Research and Evaluation of Drug-use Habits of People in North Cyprus. EURASIA Journal of Mathematics, Science and Technology Education, 11(2), 217-225. doi:10.12973/eurasia.2015.1317a

Liu, Z. L. (1993). The Diagnosis and Treatment of Lipid Metabolic abnormalities. The Journal of Chinese Cycle, 8(3), 183-185.

Ma, Z.-J. (2010). The Research of the Effect on Aerobic Exercise to Blood Lipid in Elderly. Journal of Mudanjiang Normal University (Natural Sciences Edition), 73(4), 51-52.

Perini, R., Fisher, N., Veicsteinas, A., et al. (2002). Aerobic training and cardiovascular responses at rest and during exercise in middle and older men. Med SciSports Exerc, 34, 700-708.

Seip, R. L., Angelopoulos, T. J., \& Semenkovich, C. F. (1995). Exercise includes human lipoprotein lipase gene expression in skeletal muscle bue not adipose tissue. Am J Physical, 268, 229-236.

Wang, S. T., Ceng, Y. G., Wang, A. L. (2007). The Effects of Aerobic-exercise on the Rats' GLu and GABA Neural of Cardiovascular Autonomic nerve Centre. Sports Science, 27(4), 64-66.

Wang, Z.-Z., Zhao, H.-J. (2005). The Correlation between Cardiorespiratory Function Decreasing of Overweight and Obesity Adolescent and Body Composition. Sports science, 25-27.

Yang, Q., Sui, B., Hu, Y. L., et al. (1996). On the Effects of Sports on Sebum and cardiovascular of Seniors. The Journal of ShangDong Sports Science and technology, 18(4), 8-10.

$\mathrm{Yu}, \mathrm{L}$. (2002). On the Anti-Hypertensie of Aerobic-exercise to the seniors. Chinese Clinical Rehabilitation, $5(6), 717$.

Yu, W.-B. (2008). The Analysis of the Characteristics of Aerobic Training. Journal of Mianyang Normal University, 27(11), 126-128.

Zhou, L.-Y., Wang, X., Hong, L.-D., et al. (Sep, 2008). Effects of 12-week Aerobic Exercises on Neuromuscular and Motor Functional Performance in the Elders. Chinese Journal of Rechabilitation Medicine, 23(9), 812-814.

\section{http://www.ejmste.com}

